\title{
Bladder Cancer in Iran: An Epidemiological Review
}

This article was published in the following Dove Press journal:

Research and Reports in Urology

\author{
Khadijeh Kalan Farmanfarma' \\ Neda Mahdavifar ${ }^{2}$ \\ Hamid Salehiniya ${ }^{3}$ \\ 'Department of Epidemiology, Health \\ Promotion Research Center, Zahedan \\ University of Medical Sciences, Zahedan, \\ Iran; ${ }^{2}$ Department of Biostatistics and \\ Epidemiology, School of Health, Non- \\ Communicable Diseases Research Center, \\ Sabzevar University of Medical Sciences, \\ Sabzevar, Iran; ${ }^{3}$ Social Determinants of \\ Health Research Center, Birjand University \\ of Medical Sciences, Birjand, Iran
}

Correspondence: Hamid Salehiniya Tel +989357750428

Email alesaleh70@yahoo.com
Purpose: Bladder cancer is the sixth most common cancer in Iran. Information about the epidemiological situation of the disease and its risk factors is necessary for conducting a planning program to reduce the disease. This study is conducted with the aim of evaluating the epidemiological features of bladder cancer in Iran.

Methods: This is a systematic review of the published articles in both Persian and English in international and national journals during the years 2000-2019 with key words of Iran and bladder cancer. Articles were selected from the national information database (SID, Magiran) and international databases (PubMed, Scopus, and Web of Science). Articles related to the epidemiological aspects of the disease, including incidence, prevalence, mortality, and risk factors, were evaluated. Results: The incidence rate of bladder cancer was about 1.6-115; cumulative survival rate was 0.95 , mortality rate was $0.5-0.84$ and its prevalence in the Iranian population was between $4.10 \%$ and $12.8 \%$. The risk of developing malignancy in major risk factor groups, including smoking and opium, was 6.2 (2.04-18.7), increases in protein and animal fat consumption were about 5- and 19-times higher than in the general population, respectively. In terms of occupational exposure, bus and heavy truck drivers were $11.3(1.3-92.05)$ more at risk than others.

Conclusion: Regarding the increasing trend of this disease, changes in lifestyle to reduce risk factors can be effective in decreasing the incidence and mortality rate of this malignancy.

Keywords: bladder cancer, epidemiology, Iran, incidence, risk factor

\section{Introduction}

Bladder cancer (BC) is the second most common type of urinary tract infection worldwide. Also, it is the fourth most common cancer in men and the ninth in women, ${ }^{1}$ with approximately 150,000 deaths in the world. ${ }^{1}$

The results of epidemiological studies show a wide range of genetic, anatomical, hormonal, social, and environmental factors in the development of disease. ${ }^{2}$ The incidence of this malignancy in the world is $\sim 440,000$ annually, ${ }^{3}$ the 5-year survival rates in the US and European countries were $76 \%$ and $68 \%$, respectively, which is higher in high income countries than in other countries. 4

The statistics indicate an increasing trend in prevalence of this cancer in Iran, ${ }^{5}$ with more than 70,000 new cases and 30,000 annually recorded deaths. ${ }^{6}$ Generally, $7.04 \%$ of cancers in Iran are related to bladder cancer. ${ }^{7}$ The most important risk factors associated with bladder cancer include tobacco, industrial exposures, and carcinogens such as aromatic amines, black carbon, prolonged use of arsenic or chlorine contaminated water, and family history. ${ }^{8}$

Information about the epidemiological situation of the disease and its risk factors is necessary for conducting a planning program to reduce the disease; also, there is no comprehensive conducted investigation in Iran that addresses various epidemiological 
aspects of the disease, therefore, the present study aims to examine the epidemiological aspects of bladder cancer in Iran.

\section{Methods and Materials}

This study systematically reviews articles carried out using national (SID, Magiran) and international databases (PubMed, Web of Science, Scopus) which were obtained from 2000-2019. The keywords included: "bladder cancer", "bladder neoplasm", "incidence", “epidemiology", "survival”, "mortality", "prevalence", "risk factor", and "Iran". The search was matched for each database.

Original articles that examined epidemiological aspects of the disease, including incidence, mortality, prevalence, survival, and risk factors, were included in the study. Review articles, letters to the editor, and case reports were excluded.

\section{Data Collection Process}

Summaries and full text of the articles were independently reviewed by two authors and, if relevant for the purpose of this study, required information, including the author's name, year of publication, place of study, sex of subjects, sample size, incidence, prevalence, mortality, and risk factors were extracted from each article and recorded in relevant tables. Finally, the information was presented qualitatively in the form of the tables.

\section{Results}

\section{Description of Literature Search}

A total of 723 articles were reviewed, 10 articles identified through other sources, 620 articles were duplicated, 274 articles were fully studied, 239 articles were unrelated, and finally 35 articles were included in the review (Figure 1).

\section{Mortality}

In Iran, the pattern of mortality rate reduction for bladder cancer is similar to that in high socioeconomic status

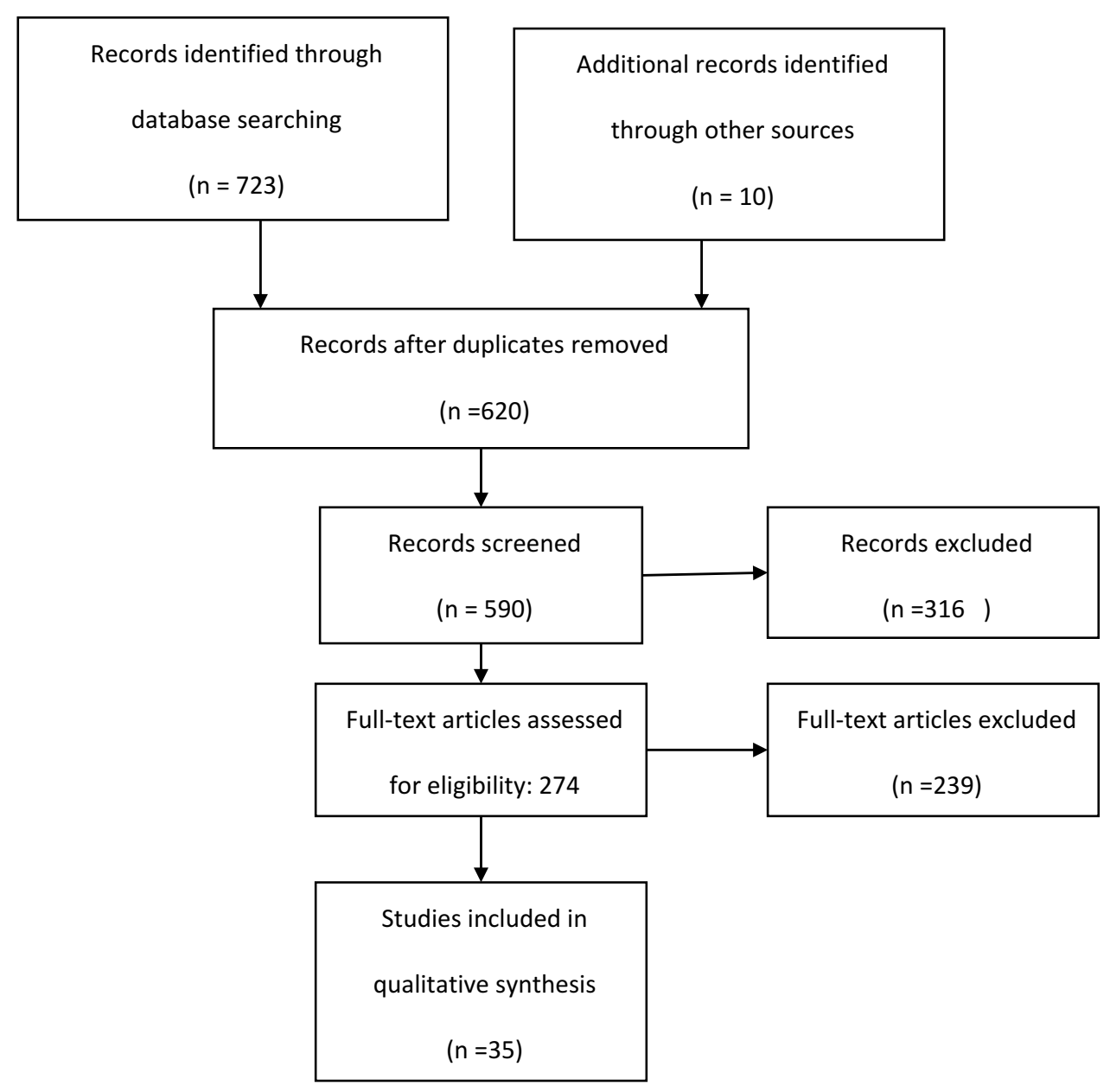

Figure I Flowchart of the included studies in the systematic review. 
countries. The mortality rate in the period of 1990-2015 decreased from 13.04 to 0.47 (Table 1).

Also, increasing in smoking among men and, at the same time, reducing the amount of cigarette consumption in women can justify a reduction in the mortality rate of bladder cancer. Meanwhile, behavioral, environmental, and metabolic changes should be considered as significant factors in reducing mortality. ${ }^{9}$

On the other hand, the efficacy and availability of diagnostic tools and therapeutic methods have been effective in reducing this malignancy. Nowadays, cystectomy is available for diagnosis and treatment in many parts of Iran, ${ }^{10,11}$ Table 1 shows the mortality of bladder cancer in Iran.

\section{Incidence}

Bladder cancer is the fifth most common cancer in Iranian men. Based on the findings of the previous studies, the standardized incidence rate in Iran was estimated to be between $1.6-115 \%$. The highest standardized incidence of age was observed in Eastern Azerbaijan (17.6) and Kerman province (15.8).

It should be noted that all previous findings in Iran reported that the incidence rate in men is more than that in women. This discrepancy is partly due to smoking habits differences among men and women, so that the incidence of malignancy in males is $1-4.8$ in southern Iran and 1-7 in other regions of country. In addition to smoking, opium consumption and occupational exposure are important risk factors which increase the malignancy rate in men. ${ }^{6}$ It should be mentioned that the incidence rate in men and women varies from 1-6 to $1-2$, and is higher in men than women, so that three quarters of new bladder cancer cases are observed in men, ${ }^{12}$ Table 2 shows the incidence of bladder cancer in Iran.

Table I The Mortality Rate of Bladder Cancer in Iran

\begin{tabular}{|c|c|c|c|c|c|c|}
\hline $\begin{array}{l}\text { First Author } \\
\text { (Year) }\end{array}$ & Province (District) & $\begin{array}{l}\text { Type of } \\
\text { Study }\end{array}$ & $\begin{array}{l}\text { Sample } \\
\text { Size }\end{array}$ & (Sex) & $\begin{array}{l}\text { Age-Standardized Mortality } \\
\text { Rate per } 100,000 \text { (ASMR) }\end{array}$ & $\begin{array}{l}\text { Death per } \\
100,000 \\
\text { People }\end{array}$ \\
\hline Babaei $(2009)^{55}$ & Ardabil & - & 4,363 & MF & 0.5 & \\
\hline \multirow[t]{4}{*}{ Mazdak $(2018)^{15}$} & \multirow[t]{4}{*}{ Isfahan } & \multirow[t]{4}{*}{$\begin{array}{l}\text { Retrospective } \\
\text { study }\end{array}$} & \multirow[t]{4}{*}{1,655} & $\begin{array}{l}\text { MF } \\
(2011-2012)\end{array}$ & 0.56 & \\
\hline & & & & $2012-2013$ & 0.74 & \\
\hline & & & & $2013-2014$ & 0.98 & \\
\hline & & & & $2014-2015$ & 0.84 & \\
\hline \multirow[t]{6}{*}{ Pishgar $(2018)^{9}$} & \multirow[t]{6}{*}{ ALLof iran } & \multirow[t]{6}{*}{ - } & \multirow[t]{6}{*}{ - } & MF (1990) & $13.04(10.04-16.95)$ & \\
\hline & & & & 1995 & $10.94(8.56-13.97)$ & \\
\hline & & & & 2000 & $5.62(4.46-7.08)$ & \\
\hline & & & & 2005 & $2.5(3.16-1.98)$ & \\
\hline & & & & 2010 & I.4 (I.78-I.I) & \\
\hline & & & & 2015 & $0.47(0.61-0.37)$ & \\
\hline Sadjadi $(2005)^{77}$ & $\begin{array}{l}\text { Golestan, Mazandaran, } \\
\text { Kerman, Ardabil Tehran }\end{array}$ & & 2,083 & MF & & 1,059 \\
\hline \multirow[t]{5}{*}{ Mahdavi $(2015)^{78}$} & \multirow[t]{5}{*}{ All of Iran } & & \multirow[t]{5}{*}{105} & MF (2006) & & 1.12 \\
\hline & & & & 2007 & & 1.09 \\
\hline & & & & 2008 & & 1.15 \\
\hline & & & & 2009 & & I.I \\
\hline & & & & 2010 & & 1.09 \\
\hline
\end{tabular}


Table 2 Incidence Rate of Bladder Cancer in Iran

\begin{tabular}{|c|c|c|c|c|c|c|c|c|}
\hline $\begin{array}{l}\text { First Author } \\
\text { (Year) }\end{array}$ & $\begin{array}{l}\text { Province } \\
\text { (District) }\end{array}$ & Type of Study & $\begin{array}{l}\text { Sample } \\
\text { Size }\end{array}$ & Sex & $\begin{array}{l}\text { ASR (Age- } \\
\text { Standardized } \\
\text { Incidence Rate) }\end{array}$ & $\begin{array}{l}\text { Incidence } \\
\text { Rate }\end{array}$ & $\begin{array}{l}\text { Incidence } \\
\text { Ratio }\end{array}$ & $\begin{array}{l}\text { Mean } \\
\pm \text { SD }\end{array}$ \\
\hline Somi $(2008)^{48}$ & East Azerbaijan & - & 4,922 & $\begin{array}{l}\text { Male } \\
\text { Female }\end{array}$ & $\begin{array}{l}15.72 \\
3.68\end{array}$ & & & \\
\hline Akbari $(2008)^{49}$ & Tehran & - & 8,147 & $\begin{array}{l}\text { Male } \\
\text { Female }\end{array}$ & & 48.3 & & \\
\hline $\begin{array}{l}\text { Rohani-Rasaf } \\
(2012)^{50}\end{array}$ & Tehran & - & - & Male & & $\begin{array}{l}\text { Sep-0I (6.3) } \\
\text { Sep-02 (8.2) } \\
\text { Sep-03 (14.2) } \\
\text { Sep-04 (15.3) }\end{array}$ & & \\
\hline $\begin{array}{l}\text { Ahmadi } \\
(2018)^{51}\end{array}$ & $\begin{array}{l}\text { Chahrmahal and } \\
\text { Bakhtiari }\end{array}$ & & 2,918 & MF & & & 0.08 & \\
\hline $\begin{array}{l}\text { Shahesmaeili } \\
(2018)^{52}\end{array}$ & Kerman & $\begin{array}{l}\text { Retrospective } \\
\text { study }\end{array}$ & 2,838 & Male & 24.70 & & & \\
\hline $\begin{array}{l}\text { Akbarzadeh } \\
(2012)^{53}\end{array}$ & Mazandaran & Cross-sectional & 310 & MF & 11.4 & & & \\
\hline $\begin{array}{l}\text { Aghajani } \\
(2016)^{54}\end{array}$ & All of Iran & $\begin{array}{l}\text { Retrospective } \\
\text { study }\end{array}$ & 301,055 & $\begin{array}{l}M \\
F\end{array}$ & $\begin{array}{l}11.25 \\
2.49\end{array}$ & & & \\
\hline Babaei $(2009)^{55}$ & Ardabil & & 4,363 & Mf & 13.1 & & & \\
\hline Basiri $(2014)^{56}$ & All of Iran & - & - & $\begin{array}{l}M(2003) \\
M(2009) \\
F(2003) \\
F(2009)\end{array}$ & $\begin{array}{l}8.35 \\
12.59 \\
1.49 \\
2.44\end{array}$ & & & \\
\hline $\begin{array}{l}\text { Haghdoost } \\
(2015)^{57}\end{array}$ & Kerman & Cross-sectional & 3,052 & MF & 15.8 & & & \\
\hline $\begin{array}{l}\text { Rafiemanesh } \\
(2018)^{58}\end{array}$ & North Khorasan & - & 2,165 & MF & 5.13 & & & \\
\hline $\begin{array}{l}\text { Rafiemanesh } \\
(2015)^{59}\end{array}$ & $\begin{array}{l}\text { Sistan and } \\
\text { Baluchistan }\end{array}$ & - & 3,535 & MF (2004) & 1.24 & & & \\
\hline Zahedi $(2015)^{60}$ & Hamadan & - & $\begin{array}{l}4,592 \\
3,175\end{array}$ & $\begin{array}{l}M(2004) \\
M(2009) \\
F(2004) \\
F(2009)\end{array}$ & $\begin{array}{l}72.9 \\
132.0 \\
48.2 \\
115.0\end{array}$ & & & \\
\hline Sadjadi $(2003)^{61}$ & Ardabil & - & $\begin{array}{l}2,072 \\
1,309\end{array}$ & $\begin{array}{l}\text { Male } \\
\text { Female }\end{array}$ & $\begin{array}{l}7.6 \\
1.8\end{array}$ & & & \\
\hline Sadjadi $(2007)^{62}$ & Kerman & - & $\begin{array}{l}3,264 \\
2,620\end{array}$ & $\begin{array}{l}M \\
F\end{array}$ & $\begin{array}{l}6.7 \\
1.6\end{array}$ & & & \\
\hline Somi $(2018)^{63}$ & East Azerbaijan & - & 6,655 & MF & 17.6 & & & \\
\hline $\begin{array}{l}\text { Salehiniya } \\
(2016)^{64}\end{array}$ & $\begin{array}{l}\text { Mazandran and } \\
\text { Golstan }\end{array}$ & $\begin{array}{l}\text { Cross- } \\
\text { sectional }\end{array}$ & $\begin{array}{l}6,177 \\
|5,23|\end{array}$ & $\begin{array}{l}M \\
F\end{array}$ & $\begin{array}{l}15.41 \\
6.77\end{array}$ & & & \\
\hline $\begin{array}{l}\text { Talaiezadeh } \\
(2013)^{65}\end{array}$ & Khouzestan & - & $|6,80|$ & $\begin{array}{l}M \\
F\end{array}$ & $\begin{array}{l}10.69 \\
4.07\end{array}$ & & & \\
\hline
\end{tabular}


Table 2 (Continued).

\begin{tabular}{|c|c|c|c|c|c|c|c|c|}
\hline $\begin{array}{l}\text { First Author } \\
\text { (Year) }\end{array}$ & $\begin{array}{l}\text { Province } \\
\text { (District) }\end{array}$ & Type of Study & $\begin{array}{l}\text { Sample } \\
\text { Size }\end{array}$ & Sex & $\begin{array}{l}\text { ASR (Age- } \\
\text { Standardized } \\
\text { Incidence Rate) }\end{array}$ & $\begin{array}{l}\text { Incidence } \\
\text { Rate }\end{array}$ & $\begin{array}{l}\text { Incidence } \\
\text { Ratio }\end{array}$ & $\begin{array}{l}\text { Mean } \\
\pm \text { SD }\end{array}$ \\
\hline $\begin{array}{l}\text { Fararouei } \\
(2015)^{66}\end{array}$ & $\begin{array}{l}\text { Kohgiluyeh and } \\
\text { Boyer-Ahmad }\end{array}$ & Cohort & 660 & MF & 6.94 & & & \\
\hline $\begin{array}{l}\text { Keyghobadi } \\
(2015)^{67}\end{array}$ & Kerman & Cross-sectional & 5,793 & $\begin{array}{l}M(2004) \\
2005 \\
2006 \\
2007 \\
2008 \\
2009\end{array}$ & $\begin{array}{l}7.73 \\
9.71 \\
9.41 \\
11.17 \\
13.20 \\
14.66\end{array}$ & & & \\
\hline $\begin{array}{l}\text { Mohagheghi } \\
(2009)^{68}\end{array}$ & Tehran & - & 26,006 & $\begin{array}{l}M \\
F\end{array}$ & $\begin{array}{l}13.3 \\
3.8\end{array}$ & & & \\
\hline $\begin{array}{l}\text { Mazdak } \\
(2018)^{15}\end{array}$ & Isfahan & $\begin{array}{l}\text { Retrospective } \\
\text { study }\end{array}$ & 1,655 & MF & $\begin{array}{l}(20 I I-2012) \\
2012-2013) \\
2013-2014) \\
2014-2015\end{array}$ & $\begin{array}{l}7.7 \\
8.1 \\
7.5 \\
9.9\end{array}$ & & \\
\hline $\begin{array}{l}\text { Masoompour } \\
(2016)^{69}\end{array}$ & Fars & - & - & & $\begin{array}{l}M(1985-1989) \\
M(1998-2002) \\
M(2007-2010) \\
F(1985-1989) \\
F(1998-2002) \\
F(2007-2010)\end{array}$ & $\begin{array}{l}7.50 \\
6.8 \\
15.60 \\
2.00 \\
1.90 \\
3.93\end{array}$ & & \\
\hline Vakili $(2014)^{70}$ & Yazd & - & 4,631 & MF & 7.5 & & & \\
\hline Yavari $(2008)^{71}$ & Tehran & & 597 & MF & & & & $\begin{array}{l}12.8 \\
\pm 61.9\end{array}$ \\
\hline \multirow[t]{6}{*}{ Rahimi $(2015)^{72}$} & Tehran & - & - & $\begin{array}{l}M \\
F\end{array}$ & $\begin{array}{l}13.3 \\
3.8\end{array}$ & & & \\
\hline & Semnan & & - & $\begin{array}{l}M \\
F\end{array}$ & $\begin{array}{l}7.16 \\
3\end{array}$ & & & \\
\hline & Golestan & & - & $\begin{array}{l}M \\
F\end{array}$ & 8.7 & & & \\
\hline & East Azerbaijan & & - & $\begin{array}{l}M \\
F\end{array}$ & $\begin{array}{l}15.72 \\
3.68\end{array}$ & & & \\
\hline & Khuzestan & & - & $\begin{array}{l}M \\
F\end{array}$ & $\begin{array}{l}10.69 \\
4.07\end{array}$ & & & \\
\hline & Shahroud & & - & $\begin{array}{l}M \\
F\end{array}$ & $\begin{array}{l}9.39 \\
3.16\end{array}$ & & & \\
\hline $\begin{array}{l}\text { Rafiemanesh } \\
(2016)^{73}\end{array}$ & QOM & Cross-sectional & 3,029 & $\begin{array}{l}M(2004) \\
2005 \\
2006 \\
2007 \\
2008\end{array}$ & $\begin{array}{l}10.46 \\
7.30 \\
6.82 \\
9.49 \\
9.80\end{array}$ & & & \\
\hline
\end{tabular}

(Continued) 
Table 2 (Continued).

\begin{tabular}{|c|c|c|c|c|c|c|c|c|}
\hline $\begin{array}{l}\text { First Author } \\
\text { (Year) }\end{array}$ & $\begin{array}{l}\text { Province } \\
\text { (District) }\end{array}$ & Type of Study & $\begin{array}{l}\text { Sample } \\
\text { Size }\end{array}$ & Sex & $\begin{array}{l}\text { ASR (Age- } \\
\text { Standardized } \\
\text { Incidence Rate) }\end{array}$ & $\begin{array}{l}\text { Incidence } \\
\text { Rate }\end{array}$ & $\begin{array}{l}\text { Incidence } \\
\text { Ratio }\end{array}$ & $\begin{array}{l}\text { Mean } \\
\pm S D\end{array}$ \\
\hline $\begin{array}{l}\text { Rafiemanesh } \\
(2018)^{74}\end{array}$ & All of Iran & Cross-sectional & 23,291 & $\begin{array}{l}F(2003) \\
2004 \\
2005 \\
2006 \\
2007 \\
2008 \\
M(2003) \\
2004 \\
2005 \\
2006 \\
2007 \\
2008\end{array}$ & $\begin{array}{l}2.12 \\
2.49 \\
2.86 \\
2.99 \\
3.32 \\
3.78 \\
8.35 \\
9.9 \\
11.3 \\
11.66 \\
13.03 \\
14.42\end{array}$ & & & \\
\hline Sadjadi $(2005)^{62}$ & $\begin{array}{l}\text { Golestan, } \\
\text { Mazandaran, } \\
\text { Kerman, Ardabil } \\
\text { Tehran }\end{array}$ & - & 51,000 & $M$ & 8.0 & & & \\
\hline $\begin{array}{l}\text { Enayatrad } \\
(2016)^{75}\end{array}$ & All of Iran & - & $\begin{array}{l}41,169 \\
32,898\end{array}$ & $\begin{array}{l}M(2004) \\
2005 \\
2006 \\
2007 \\
2009 \\
F(2004) \\
2005 \\
2006 \\
2007 \\
2009\end{array}$ & $\begin{array}{l}11.3 \\
11.7 \\
13.0 \\
14.4 \\
12.6 \\
2.9 \\
2.9 \\
3.3 \\
3.8 \\
3.3\end{array}$ & & & \\
\hline Koohi $(2015)^{76}$ & All of Iran & Cross-sectional & 27,898 & $\begin{array}{l}\text { MF (2003) } \\
2004 \\
2005 \\
2006 \\
2007 \\
2008 \\
2009\end{array}$ & $\begin{array}{l}4.69 \\
4.70 \\
4.71 \\
4.71 \\
4.63 \\
4.48 \\
4.49\end{array}$ & & & \\
\hline
\end{tabular}

\section{Survival}

The average survival rate was $\sim 24.2$ months in the country. The survival time is related to only two variables of the place of living and the type of disease, given that the economic, social, and cultural structure of the developing countries is approaching advanced countries, it can be expected that the incidence of bladder cancer in Iranian women will increase in the future, since the 5-year survival rate of bladder cancer in women is 6 years less than that of men. ${ }^{13}$ Also, the cumulative survival rate in Iran was $0.95 \%$, indicating that the survival rate of $\mathrm{BC}$ in Iranian patients is lower than in other areas.
However, the effect of early diagnosis based on updated and effective methods in advanced countries should not be underestimated. In other words, this difference can be due to the existence of a decision-making process or lead time bias. ${ }^{14}$ Table 3 shows the survival rate of bladder cancer in Iran.

\section{Prevalence}

Based on previous studies, the prevalence rate in this Iranian population was 33.2 per 100,000 people (Table 4 ). This rate in men is 4.8-times higher than that in women. ${ }^{15}$ The high prevalence of this malignancy can reflect the patterns of 
Table 3 The Survival Rate Associated with Bladder Cancer in Iran

\begin{tabular}{|l|l|l|l|l|l|l|l|}
\hline $\begin{array}{l}\text { First Author } \\
\text { (Year) }\end{array}$ & $\begin{array}{l}\text { Province } \\
\text { (District) }\end{array}$ & Type of Study & Sex & $\begin{array}{l}\text { Sample } \\
\text { Size }\end{array}$ & $\begin{array}{l}\text { Average } \\
\text { Survival Rate }\end{array}$ & Survival Rate & $\begin{array}{l}\text { Cumulative } \\
\text { Survival Rate }\end{array}$ \\
\hline Ahmad (2003) $)^{13}$ & Fars & Cohort Historical & MF & 76 & $24.2(18.3-30.1)$ & - & - \\
\hline $\begin{array}{l}\text { Mohammadbeigi } \\
(201)^{14}\end{array}$ & Fars & $\begin{array}{l}\text { Retrospective } \\
\text { study }\end{array}$ & MF & 514 & - & $\begin{array}{l}\text { One-year } \\
\text { survival }=0.9 \\
3 \text { years }=0.7 \\
5 \text { years }=0.6 \\
10 \text { years }=0.2\end{array}$ & 0.9582 \\
\hline $\begin{array}{l}\text { Vahedian- } \\
\text { Ardakani (2018) }\end{array}$ & Yazd & $\begin{array}{l}\text { Retrospective } \\
\text { cohort study }\end{array}$ & MF & 340 & - & - & $\begin{array}{l}\text { One-year survival }=91 \\
3 \text { years }=58 \\
5 \text { years }=51.4\end{array}$ \\
\hline
\end{tabular}

Table 4 The Prevalence of Bladder Cancer in Iran

\begin{tabular}{|c|c|c|c|c|c|c|}
\hline $\begin{array}{l}\text { First Author } \\
\text { (Year) }\end{array}$ & Province (District) & Type of Study & $\begin{array}{l}\text { Sample } \\
\text { Size }\end{array}$ & Sex & Prevalence & $\begin{array}{l}5 \text { Year } \\
\text { Prevalence }\end{array}$ \\
\hline Nikfarjam $(2014)^{79}$ & Mashhad & - & 4606 & MF & 4.10 & \\
\hline Farahmand $(2009)^{80}$ & Fars & - & 1440 & $\begin{array}{l}\operatorname{Mf}(2002) \\
2003 \\
2004 \\
2005 \\
2006\end{array}$ & $\begin{array}{l}173 \\
201 \\
207 \\
351 \\
314\end{array}$ & \\
\hline Mazdak $(2018)^{15}$ & Isfahan & $\begin{array}{l}\text { Retrospective } \\
\text { study }\end{array}$ & 1655 & $\begin{array}{l}\text { MF } \\
(2011-2015)\end{array}$ & 33.2 & \\
\hline Rahimi $(2015)^{73}$ & Kermanshah & - & 7695 & MF & 12.8 & \\
\hline Roshani $(2016)^{81}$ & All of iran & Cross-sectional & 75,500 & MF & 9.6 & \\
\hline Sadjadi $(2005)^{77}$ & $\begin{array}{l}\text { Golestan, Mazandaran, Kerman, } \\
\text { Ardabil Tehran }\end{array}$ & - & 2083 & MF & - & 5970 \\
\hline
\end{tabular}

smoking and environmental carcinogens. ${ }^{16}$ Table 4 shows the prevalence of bladder cancer in Iran.

\section{Risk Factors}

\section{Smoking}

Researchers report the high prevalence of smoking and opium consumption in the Iranian studied population. ${ }^{17}$ The chance of malignancy in Iran in smokers and opium users is $6.2(2.04-18.7)$ and (1.8-7.8), being $8.3 \%$ times higher than those who only smoke (Table 5).

The risk associated with cigarette smoking is considered to be an important disposal factor for the incidence of bladder cancer of $50 \%{ }^{3}$

The use of opium in Iran over a hundred years is one of the most commonly used drugs. The number of addicts increases by $8 \%$ every year, ${ }^{18}$ also the largest growth is observed in northern and southern parts of Iran. ${ }^{19,20}$ As surveys show, opium consumption alone increases the chance of BC by 4.60-times (Table 5).

\section{Gender and Age}

The results reveal the differences in the incidence and survival rate among men and women, as these are higher in the male population. ${ }^{3}$ In a meta-analysis study, the lowest incidence rate in Iranian women is estimated at 0.4 per 100,000 people. ${ }^{5}$ Factors such as differences in access to healthcare, late diagnosis, occupational exposure, pattern of Cigarette smoking, and the presence of steroid hormone in the two sexes ${ }^{3}$ are the reasons for these discrepancies. Increasing age as a risk factor plays an important role in the incidence of BC. The results of studies in Iran showed that the mean age of patients with BC in Iran was $65.1,61.9$, and 55.5 years, ${ }^{6}$ so that $47 \%$ of new cases in Iran are over 65 years old. ${ }^{21}$ 
Table 5 Risk Factors Associated with Bladder Cancer in Iran

\begin{tabular}{|c|c|c|c|c|c|c|c|c|c|}
\hline $\begin{array}{l}\text { First Author } \\
\text { (Year) }\end{array}$ & $\begin{array}{l}\text { Province } \\
\text { (District) }\end{array}$ & $\begin{array}{l}\text { Type of } \\
\text { Study }\end{array}$ & Sample Size & Sex & Risk Factors & OR & $\begin{array}{l}\text { Mean (SD; } \\
\text { Min, Max) }\end{array}$ & $\mathbf{N}(\%)$ & $\begin{array}{l}\text { Median } \\
95 \% \mathrm{Cl}\end{array}$ \\
\hline Akbari $(2015)^{18}$ & Fars & Case-control & $\begin{array}{l}198 \text { (case) } \\
396 \text { (control) }\end{array}$ & $\begin{array}{l}\text { Male } \\
\text { Female }\end{array}$ & $\begin{array}{l}\text { Opium } \\
\text { Tobacco } \\
\text { Alcohol }\end{array}$ & $\begin{array}{l}3.9(1.3-12.0) \\
2.5(1.3-4.8) \\
2.9(1.2-7.1)\end{array}$ & & & \\
\hline Aminian $(2014)^{36}$ & Tehran & Case-control & $\begin{array}{l}160 \text { (case) } \\
160 \text { (control) }\end{array}$ & Male & $\begin{array}{l}\text { Occupational risk: } \\
\text { I-bus and truck driving } \\
\text { 2-mechanics, working in } \\
\text { refinery and Petrochemical, } \\
\text { plastic } \\
\text { 3-Textile industry, Spinning, } \\
\text { Hosiery } \\
\text { 4-House Keeping } \\
\text { 5-barberry, painting, Dye } \\
\text { Manufacturing } \\
\text { 6-Shoe-making, production, } \\
\text { seat cover, Embroidered bag, } \\
\text { Repairing shoes } \\
\text { 7-welding, metal working, } \\
\text { plumbing, turning } \\
\text { 8-driving } \\
\text { 9-office job } \\
\text { 10-carpentry, Mosaic work, } \\
\text { cabinet making }\end{array}$ & $\begin{array}{l}3.62(1.04-12.89) \\
2.71(1.03-7.59) \\
2.23(0.70-7.15) \\
1.79(0.90-3.67) \\
1.81(0.69-4.83) \\
1.12(0.34-4.57) \\
\\
2.46(0.99-6.28) \\
1.57(0.69-3.49) \\
1.15(0.65-2.0) \\
1.14(0.27-4.07)\end{array}$ & & & \\
\hline Ahmadi $(2012)^{6}$ & Mazandaran & $\begin{array}{l}\text { Cross- } \\
\text { sectional }\end{array}$ & 112 & MF & $\begin{array}{l}\text { Opium } \\
\text { Tobacco } \\
\text { History of cancer in family }\end{array}$ & & & $\begin{array}{l}21.4 \\
45.5 \\
22.32\end{array}$ & \\
\hline Khoubi $(2013)^{34}$ & Isfahan & Case-control & $\begin{array}{l}300 \text { (case) } \\
500 \text { (control) }\end{array}$ & & $\begin{array}{l}\text { Health professionals } \\
\text { Petroleum and natural gas } \\
\text { refining plant operators } \\
\text { Clerks } \\
\text { Domestic housekeepers } \\
\text { Sales workers } \\
\text { Shop assistants } \\
\text { Skilled agricultural, forestry, } \\
\text { and fishery workers } \\
\text { Animal producers } \\
\text { Building and related trades } \\
\text { workers, excluding } \\
\text { electricians } \\
\text { Carpenters and joiners } \\
\text { Painters and related workers } \\
\text { Sheet and structural metal } \\
\text { workers, molders and welders } \\
\text { Food processing and related } \\
\text { trades workers } \\
\text { Car, van, and motorcycle } \\
\text { drivers } \\
\text { Heavy truck and bus drivers } \\
\text { Mining and construction } \\
\text { laborers }\end{array}$ & $\begin{array}{l}0.4(0.04-4.80) \\
0.4(0.18-1.50) \\
0.2(0.11-2.49) \\
5.9(2.61-11.62) \\
2.8(0.81-2.01) \\
3.4(0.91-7.21) \\
6.0(2.32-15.72) \\
2.5(0.70-9.04) \\
3.8(1.33-3.21) \\
2.9(0.77-12.13) \\
1.8(0.20-4.21) \\
6.0(2.84-14.08) \\
1.0(0.22-3.52) \\
0.6(0.07-5.60) \\
11.3(1.32-92.50) \\
1.2(0.30-4.41)\end{array}$ & & & \\
\hline Hosseini $(2010)^{37}$ & Tehran & Case-control & $\begin{array}{l}179 \text { (case) } \\
179 \text { (control) }\end{array}$ & & Opium & $4.60(3.53-6.28)$ & & & \\
\hline
\end{tabular}

(Continued) 
Table 5 (Continued).

\begin{tabular}{|c|c|c|c|c|c|c|c|c|c|}
\hline $\begin{array}{l}\text { First Author } \\
\text { (Year) }\end{array}$ & $\begin{array}{l}\text { Province } \\
\text { (District) }\end{array}$ & $\begin{array}{l}\text { Type of } \\
\text { Study }\end{array}$ & Sample Size & Sex & Risk Factors & OR & $\begin{array}{l}\text { Mean (SD; } \\
\text { Min, Max) }\end{array}$ & $\mathbf{N}(\%)$ & $\begin{array}{l}\text { Median } \\
95 \% \mathrm{Cl}\end{array}$ \\
\hline $\begin{array}{l}\text { Shakhssalim } \\
(2010)^{7}\end{array}$ & $\begin{array}{l}\text { Tehran, } \\
\text { Khorasan, } \\
\text { Khoozestan, } \\
\text { Isfahan and } \\
\text { East } \\
\text { Azarbayjan }\end{array}$ & Case-control & $\begin{array}{l}692 \text { (case) } \\
692 \text { (control) }\end{array}$ & MF & $\begin{array}{l}\text { Opium } \\
\text { Hair dye } \\
\text { Excessive analgesics use } \\
\text { Sausage } \\
\text { Ham } \\
\text { Smoked fish } \\
\text { Can } \\
\text { Pickles } \\
\text { Fruits } \\
\text { Vegetables }\end{array}$ & $\begin{array}{l}2.57(1.55-4.26) \\
1.99(1.04-3.82) \\
1.41(1.02-1.94) \\
0.98(0.70-1.34) \\
1.24(0.87-1.78) \\
0.78(0.50-1.20) \\
0.80(0.60-1.07) \\
0.93(0.88-0.99) \\
0.97(0.94-1.00) \\
0.93(0.89-0.97)\end{array}$ & & & \\
\hline Farzaneh $(2017)^{38}$ & Yazd & Case-control & $\begin{array}{l}200 \text { (case) } \\
200 \text { (control) }\end{array}$ & MF & $\begin{array}{l}\text { Chronic or recurrent } \\
\text { urinary } \\
\text { History of chronic or } \\
\text { recurrent urinary tract } \\
\text { infection 2-3 years } \\
\text { Analgesic use } \\
\text { History of analgesic use } \geq 4 \\
\text { years } \\
\text { Irradiation to pelvis } \\
\text { History of irradiation to } \\
\text { pelvis } \geq 4 \text {-times } \\
\text { Kidney and bladder stones } \\
\text { History of kidney and } \\
\text { bladder stones } \geq 2 \text { years } \\
\text { History of diabetes } \\
\text { History of other kidney } \\
\text { diseases } \\
\text { History of hypertension } \\
\text { History of smoking }\end{array}$ & $\begin{array}{l}5.92(3.47-10.09) \\
8.18(2.18-30.69) \\
2.21(1.30-3.75) \\
11.81(2.83-49.18) \\
1.69(1.10-2.58) \\
12.68(3.51-45.76) \\
3.44(2.06-5.75) \\
8.07(2.48-26.22) \\
2.15(1.29-3.59) \\
2.84(1.8-4.46) \\
1.81(1.08-3.02) \\
2.87(1.47-5.59)\end{array}$ & & & \\
\hline Ghadimi $(2015)^{39}$ & Kurdistan & Case-control & 152 & MF & $\begin{array}{l}\text { Ex-smoker } \\
\text { Current smoker } \\
\text { Occupational risk: } \\
\text { Driver } \\
\text { Loom } \\
\text { Carpenter } \\
\text { Cleaner } \\
\text { Painter } \\
\text { Metal } \\
\text { Construction worker } \\
\text { Farmer } \\
\text { Governmental employee } \\
\text { BMI>25 }\end{array}$ & $\begin{array}{l}2.549(1.287-5.048) \\
1.714(0.824-3.563) \\
5.134(0.593-44.47) \\
3.039 \\
(0.312-29.551) \\
0.688(0.376-1.258) \\
1.342(0.295-6.101) \\
10.629 \\
(1.343-84.093) \\
0.814(0.434-1.528) \\
0.819(0.513-1.308) \\
1.128(0.571-2.228) \\
1.041(0.657-1.649)\end{array}$ & & & \\
\hline Lotfi $(2016)^{40}$ & Yazd & Case-control & $\begin{array}{l}200 \text { (case) } \\
200 \text { (control) }\end{array}$ & MF & $\begin{array}{l}\text { Cigarette smoking } \\
\text { Opium History } \\
\text { History of Hubble bubble } \\
\text { and pipe } \\
\text { History of alcohol } \\
\text { Oil Animal } \\
\text { Fried food (per week) } \\
\text { Red Meat }>4\end{array}$ & $\begin{array}{l}3.16(2.04-4.9) \\
3.01(1.73-5.23) \\
2.63(1.48-4.64) \\
\\
2.57(1.04-6.35) \\
3.19(1.64-6.21) \\
4.02(2.32-6.95) \\
5.61(3.02-10.41)\end{array}$ & & & \\
\hline
\end{tabular}

(Continued) 
Table 5 (Continued).

\begin{tabular}{|c|c|c|c|c|c|c|c|c|c|}
\hline $\begin{array}{l}\text { First Author } \\
\text { (Year) }\end{array}$ & $\begin{array}{l}\text { Province } \\
\text { (District) }\end{array}$ & $\begin{array}{l}\text { Type of } \\
\text { Study }\end{array}$ & Sample Size & Sex & Risk Factors & OR & $\begin{array}{l}\text { Mean (SD; } \\
\text { Min, Max) }\end{array}$ & N (\%) & $\begin{array}{l}\text { Median } \\
95 \% \mathrm{Cl}\end{array}$ \\
\hline Mohseni $(2004)^{41}$ & Tehran & Case-control & 185 & MF & $\begin{array}{l}\text { Smoking in the patients with } \\
\text { high-grade tumors }\end{array}$ & $15.9(6.7-36.9)$ & & & \\
\hline Motamed $(2018)^{42}$ & Fars & Retrospective & 756 & MF & $\begin{array}{l}\text { Hookah consumption } \\
\text { Hypertension } \\
\text { Gender }\end{array}$ & $\begin{array}{l}1.44(1.01-2.1) \\
1.36(1.00-1.86) \\
1.46(0.99-2.15)\end{array}$ & & & \\
\hline Mirsafa $(2015)^{43}$ & Tehran & Case-control & $\begin{array}{l}55 \text { (case) } \\
110 \text { (control) }\end{array}$ & & $\begin{array}{l}\text { Animal fat } \\
\text { FAT } \\
\text { Junk foods } \\
\text { Organ meat } \\
\text { Processed meat } \\
\text { Sweets }\end{array}$ & $\begin{array}{l}19.76 \\
12.92 \\
8.1 \\
5.47 \\
5.34 \\
3.62\end{array}$ & & & \\
\hline $\begin{array}{l}\text { Jafari-Koshki } \\
(2017)^{44}\end{array}$ & All of Iran & - & 17,792 & & $\begin{array}{l}\text { BMI>25 (2006) } \\
\text { BMI>25 (2007) } \\
\text { BMI>25 (2008) } \\
\text { Cigarette (2006) } \\
\text { Cigarette (2007) } \\
\text { Cigarette (2008) } \\
\text { Fruit (2006) } \\
\text { Fruit (2007) } \\
\text { Fruit (2008) } \\
\text { Vegetables (2006) } \\
\text { Vegetables (2007) } \\
\text { Vegetables (2008) } \\
\text { Low activity (2006) } \\
\text { Low activity (2007) } \\
\text { Low activity (2009) }\end{array}$ & & $\begin{array}{l}43.28 \pm 6.65 \\
44.59 \pm 6.18 \\
42.99 \pm 4.93 \\
11.94 \pm 1.92 \\
12.75 \pm 1.74 \\
11.64 \pm 1.94 \\
1.13 \pm 0.24 \\
1.32 \pm 0.27 \\
1.42 \pm 0.40 \\
1.25 \pm 0.38 \\
1.38 \pm 0.42 \\
1.41 \pm 0.44 \\
30.67 \pm 7.46 \\
34.88 \pm 8.38 \\
38.30 \pm 8.15\end{array}$ & & \\
\hline Mahaki $(2011)^{45}$ & All of Iran & - & 62,037 & MF & $\begin{array}{l}\text { Smoking } \\
\text { Socioeconomic }\end{array}$ & & & & $\begin{array}{l}0.97 \\
(0.43-2.11) \\
0.92 \\
(0.45-1.88)\end{array}$ \\
\hline Islami $(2007)^{46}$ & Tehran & Case-control & $\begin{array}{l}147 \text { (case) } \\
39 \text { (control) }\end{array}$ & MF & HPV virusei & & & $\begin{array}{l}34.7 \\
7.6\end{array}$ & \\
\hline
\end{tabular}

\section{Lifestyle}

High consumption of alcohol, ${ }^{22,23}$ low consumption of vegetables and fruits, and low levels of drinking water, ${ }^{24}$ also, consumption of water containing halo methane chloride, ${ }^{16,25}$ Schistosoma infection (9-25\%), and occupational exposure to aromatic amines, ${ }^{26}$ Chronic bladder infection, history of diabetes, and metabolic syndrome ${ }^{27}$ are associated with the risk of developing BC.

In this study, the association between alcohol consumption and malnutrition was reported to be around $9-57 \%$ (Table 1), the majority of studies in the world also refer to the relationship between alcohol and BC. ${ }^{28}$ Family history, as an important factor, Increases the risk of malignancy by $22.32 \%$ (Table 5), which itself indicates the role of genetics in the occurrence of this malignancy. ${ }^{29}$
It should be noted that obesity increases the risk of $\mathrm{BC}$ by 1.04-fold (Table 5). Since the relationship between obesity and type 2 diabetes is absolutely known, type 2 diabetes is associated with insulin resistance and serum IGF-1 levels, as in patients with BC, IGF-1 levels are significantly higher. On the other hand, diabetes is associated with an increased risk of bladder infection, which causes the higher incidence of $\mathrm{BC} .^{30}$ In the present study, diabetes also increased the risk of BC by 2.15-times (Table 5).

Studies have shown that there is a relationship between diet and cancers. ${ }^{31}$ In this study, carbohydrates are associated with a $62 \%$ risk of $\mathrm{BC}$ incidence (Table 5 ).

Meanwhile, taking green and yellow fruits and vegetables can have a protective effect on this disease. Fruits and vegetables reduce the risk due to the presence of alpha-beta- 
carotene, lycopene, lutein, and vitamins A, E, C, and F. ${ }^{32}$ In the investigated studies in Iran, the protective effect of fruits and vegetables was $\mathrm{OR}=0.97 \quad(95 \% \mathrm{CI}=0.94-1.00)$ and $\mathrm{OR}=0.93$ (95\% CI=0.99-0.99), respectively.

In contrast, a high intake of meat and fat is associated with the risk of this malignancy. ${ }^{32}$ As a result, the consumption of animal protein increases the risk of infection in Iran by about 5 , and with animal fat the risk is elevated by $\sim 19$-times (Table 5).

In a study, $34.7 \%$ of the patients with $\mathrm{BC}$ were found to have an HPV virus, while in the control group the infection was $7.6 \%$, indicating a relationship between the HPV virus and the incidence of BC (Table 5). In a metaanalysis, HPV outbreak worldwide was $16.88 \%,{ }^{33}$ which is much lower than that reported in Iran. The difference can be due to genetic and environmental factors, sexual behavior, and ethnic and cultural differences in different parts of the world, including Iran. ${ }^{33}$

\section{Occupational Exposure}

In total, $20 \%$ of the causes of bladder cancer are due to occupational exposure. ${ }^{34}$ Based on previous research, the chance of developing $\mathrm{BC}$ among bus and heavy drivers is 11.3 (1.3-92.05), in farmers, fisheries, and forestry, it is 6.0 (2.32-15.72), and among metalworkers and welders it is 6.0 (2.84-14.08), these all being higher than others, such as carpenters, at $0.68(0.35-1.25)$ (Table 5).

On the other hand, evidence suggests that construction workers and those who deal with chemical substances are more likely to develop an invasive type of malignancy. ${ }^{35}$ The carcinogenic effects of occupational exposure are more due to the presence of benzidine, ortho-toluidine, 2- naphylamine, 4-aminobiphenyl, and 4,4'-methylene-bis (2-chloroaniline) (MBOCA). ${ }^{16}$

\section{Conclusion}

The aim of this study was to determine the epidemiological aspects of bladder cancer in Iran. BC is the sixth most common cancer in the Iranian population, with a standardized incidence of 10.92 years for men and 2.80 for women. The incidence rate ranged from 1.6-115, the cumulative survival rate was 0.95 , mortality ranged from $0.5-0.84$, and the prevalence ranged from $4.10-12.8 \%$. It seems that changes in Iranian lifestyle, as well as regular screening, can be effective in reducing the incidence and severity of this malignancy. Smoking and opium, consumption of red meat more than 4 times a week, animal fat, and occupational exposure such as bus and motor carriers are also considered risk factors for this cancer.

\section{Disclosure}

The authors report no conflicts of interest in this work.

\section{References}

1. Afsharmoghadam N, Haghighatian Z, Mazdak H, Mirkheshti N, Koushki RM, Alavi SA. Concentration-dependent effects of curcumin on 5-fluorouracil efficacy in bladder cancer cells. Asian Pac J Cancer Prev. 2017;18(12):3225-3230. doi:10.22034/APJCP.2017.18.12.3225

2. Aghaalikhani N, Rashtchizadeh N, Shadpour P, Allameh A, Mahmoodi M. Cancer stem cells as a therapeutic target in bladder cancer. J Cell Physiol. 2019;234(4):3197-3206. doi:10.1002/jcp.26916

3. Cumberbatch MGK, Jubber I, Black PC, et al. Epidemiology of bladder cancer: a systematic review and contemporary update of risk factors in 2018. Eur Urol. 2018;74(6):784-795. doi:10.1016/j. eururo.2018.09.001

4. Diet N Physical activity and bladder cancer; 2015. Available from: https://www.cancerpreventionscotland.org.uk/research/diet-nutritionphysical-activity-and-bladder-cancer. Accessed February 3, 2020.

5. Hassanipour S, Delam H, Fathalipour M, et al. The incidence of bladder cancer in Iran: a systematic review and meta-analysis. WCRJ. 2019;6:e1222.

6. Ahmadi M, Ranjbaran H, Amiri MM, et al. Epidemiologic and socioeconomic status of bladder cancer in Mazandaran Province, Northern Iran. Asian Pac J Cancer Prev. 2012;13(10):5053-5056. doi:10.7314/ APJCP.2012.13.10.5053

7. Shakhssalim N, Hosseini SY, Basiri A, Eshrati B, Mazaheri M, Soleimanirahbar A. Prominent bladder cancer risk factors in Iran. Asian Pac J Cancer Prev. 2010;11(3):601-606.

8. Wong MC, Fung FD, Leung C, Cheung WW, Goggins WB, Ng C. The global epidemiology of bladder cancer: a join point regression analysis of its incidence and mortality trends and projection. Sci Rep. 2018;8(1):1129.

9. Pishgar F, Amini E, Gohari K, et al. National and subnational mortality of urological cancers in Iran, 1990-2015. Asia Pac J Clin Oncol. 2019;15(2):e43-e48. doi:10.1111/ajco.13074

10. Asgari M, Safarinejad M, Shakhssalim N, Soleimani M, Shahabi A, Amini E. Quality of life after radical cystectomy for bladder cancer in men with an ileal conduit or continent urinary diversion: a comparative study. Urol Ann. 2013;5(3):190. doi:10.4103/0974-7796.115747

11. Tabibi A, Simforoosh N, Parvin M, et al. Predictive factors for prostatic involvement by transitional cell carcinoma of the bladder. Urol J. 2011;8(1):43-47.

12. Ferlay J. Estimated cancer incidence, mortality and prevalence worldwide in 2012. GLOBOCAN. 2012. Available from: https://publications.iarc.fr > Databases > Iarc-Cancerbases > GLOBOCAN-2. Accessed February 3, 2020.

13. Ahmad E, Ghasem Khani O, Dastoori P. Recurrence rate and prognostic factors of superficial bladder cancer in shiraz shahid faghihi hospital during the years 1998-2001. J Adv Med Biomed Res. 2003;11(42):1-7.

14. Mohammadbeigi A, Zadeh AR. Application of life table in survival analysis of patients with bladder cancer. ZJRMS. 2011;13(3):25-29.

15. Mazdak H, Tolou-Ghamari Z. Preliminary study of prevalence for bladder cancer in Isfahan Province, Iran. Arab J Urol. 2018;16 (2):206-210. doi:10.1016/j.aju.2017.11.017

16. Cumberbatch MG, Noon AP. Epidemiology, aetiology and screening of bladder cancer. Transl Androl Urol. 2019;8(1):5. doi:10.21037/ tau.2018.09.11

17. Aliasgari M, Kaviani A, Gachkar L, Hosseini-Nassab S. Is bladder cancer more common among opium addicts? Urol J. 2009;1 (4):253-2535. 
18. Akbari M, Naghibzadeh TA, Khanjani N, et al. Opium as a risk factor for bladder cancer: a population-based case-control study in Iran. Arch Iran Med. 2015;18(9):567-571. doi:0151809/AIM.004

19. Ziaaddini H, Ziaaddini MR. The household survey of drug abuse in Kerman, Iran. $J$ Appl Sci. 2005;5(2):380-382. doi:10.3923/jas. 2005.380.382

20. Nakhaee N, Divsalar K, Meimandi MS, Dabiri S. Estimating the prevalence of opiates use by unlinked anonymous urine drug testing: a pilot study in Iran. Subst Use Misuse. 2008;43(3-4):513-520. doi:10.1080/10826080701772348

21. Yavari P, Sadrolhefazi B, Mohagheghi M, Mehrazin R. A descriptive retrospective study of bladder cancer at a hospital in Iran (1973-2003). Asian Pac J Cancer Prev. 2009;10(4):681-684.

22. Zaitsu M, Nakamura F, Toyokawa S, et al. Risk of alcohol consumption in bladder cancer: case-control study from a nationwide inpatient database in Japan. Tohoku J Exp Med. 2016;239(1):9-15. doi:10.1620/tjem.239.9

23. Botteri E, Ferrari P, Roswall N, et al. Alcohol consumption and risk of urothelial cell bladder cancer in the European prospective investigation into cancer and nutrition cohort. Int J Cancer. 2017;141 (10):1963-1970. doi:10.1002/ijc.v141.10

24. Di Maso M, Bosetti C, Taborelli M, et al. Dietary water intake and bladder cancer risk: an Italian case-control study. Cancer Epidemiol. 2016;45:151-156. doi:10.1016/j.canep.2016.09.015

25. Temraz S, Haibe Y, Charafeddine M, Saifi O, Mukherji D, Shamseddine A. The unveiling of a new risk factor associated with bladder cancer in Lebanon. BMC Urol. 2019;19(1):16. doi:10.1186/ s12894-019-0445-9

26. Burger M, Catto J, Dalbagni G, et al. Epidemiology and risk factors of urothelial bladder cancer. Eur Urol. 2013;63:234-241. doi:10.1016/j. eururo.2012.07.033

27. Bravi F, Spei M-E, Polesel J, et al. Mediterranean diet and bladder cancer risk in Italy. Nutrients. 2018;10(8):1061. doi:10.3390/nu10081061

28. Pelucchi C, Galeone C, Tramacere I, et al. Alcohol drinking and bladder cancer risk: a meta-analysis. Ann Oncol. 2011;23(6):1586-1593. doi:10. 1093/annonc/mdr460

29. Murta-Nascimento C, Silverman DT, Kogevinas M, et al. Risk of bladder cancer associated with family history of cancer: do low-penetrance polymorphisms account for the increase in risk? Cancer Epidemiol Biomarkers Prev. 2007;16(8):1595-1600. doi:10.1158/1055-9965.EPI-06-0743

30. Qin Q, Xu X, Wang X, Zheng X-Y. Obesity and risk of bladder cancer: a meta-analysis of cohort studies. Asian Pac J Cancer Prev. 2013;14(5):3117-3121. doi:10.7314/APJCP.2013.14.5.3117

31. Farma KKF, Jalili Z, Zareban I, Pour MS. Effect of education on preventive behaviors of breast cancer in female teachers of guidance schools of Zahedan city based on health belief model. J Educ Health Promot. 2014;3.

32. Piyathilake C. Dietary factors associated with bladder cancer. Invest Clin Urol. 2016;57(Suppl 1):S14-S25. doi:10.4111/icu.2016.57.S1.S14

33. Li N, Yang L, Zhang Y, Zhao P, Zheng T, Dai M. Human papillomavirus infection and bladder cancer risk: a meta-analysis. $J$ Infect Dis. 2011;204(2):217-223. doi:10.1093/infdis/jir248

34. Khoubi J, Pourabdian S, Mohebbi I, Tajvidi M, Zaroorian O, Giahi O. Association between the high risk occupations and bladder cancer in Iran: a case-control study. Int J Occup Med Environ Health. 2013;26(2):205-213. doi:10.2478/s13382-013-0103-5

35. Noon AP, Martinsen JI, Catto JW, Pukkala E. Occupation and bladder cancer phenotype: identification of workplace patterns that increase the risk of advanced disease beyond overall incidence. Eur Urol Focus. 2018;4(5):725-730. doi:10.1016/j.euf.2016.06.014

36. Aminian O, Saburi A, Mohseni H, Akbari H, Chavoshi F, Akbari H. Occupational risk of bladder cancer among Iranian male workers. Urol Ann. 2014;6(2):135. doi:10.4103/0974-7796.130643

37. Hosseini SY, Safarinejad MR, Amini E, Hooshyar H, editors. Opium consumption and risk of bladder cancer: a case-control analysis. Urol Oncol. 2010;28(6):610-616. doi:10.1016/j.urolonc.2008.10.016
38. Farzaneh F, Mehrparvar AH, Lotfi MH. Occupations and the risk of bladder cancer in Yazd Province: a Case-Control study. Int J Occup Environ Med. 2017;8:191-198. doi:10.15171/ijoem.2017.1085

39. Ghadimi T, Gheitasi B, Nili S, Karimi M, Ghaderi E. Occupation, smoking, opium, and bladder cancer: a case-control study. South Asian J Cancer. 2015;4(3):111. doi:10.4103/2278-330X.173174

40. Lotfi M, Bagheri M, Mosleh-Shirazi M, Faghihi R, BaradaranGhahfarokhi M. Evaluation of the changes in the shape and location of the prostate and pelvic organs due to bladder filling and rectal distension. Iran Red Crescent Med J. 2011;54:26-67.

41. Mohseni MG, Zand S, Aghamir SMK. Effect of smoking on prognostic factors of transitional cell carcinoma of the bladder. Urol J. 2009;1(4):250-252.

42. Motamed S, Aminsharifi A, Vardanjani H, Salehi A. Bladder cancer in southwestern Iran: histopathologic and clinical features. Pak J Med Health Sci. 2018;12(2):884-888.

43. Mirsafa F, Abdolahi M, Houshiar Rad A. The relationship between food intake and bladder cancer: a Case Control study. YUMSJ. 2015;19(10):828-840.

44. Jafari-Koshki T, Arsang-Jang S, Mahaki B. Bladder cancer in Iran: geographical distribution and risk factors. Iran $J$ Cancer Prev. 2017;10:2.

45. Mahaki B, Mehrabi Y, Kavousi A, et al. Multivariate disease mapping of seven prevalent cancers in Iran using a shared component model. Asian Pac J Cancer Prev. 2011;12(9):2353-2358.

46. Eslami G, Golshani M, Rakhshan M, Fallah F, Goudarzi H, Taghavi A. Surveying human papiloma virus (HPV) in patients with bladder cancer by PCR. Res Med. 2007;31(4):339-343.

47. Vahedian-Ardakani H-A, Moghimi M, Shayestehpour M, Doosti M, Alimohammadi F. Survival rate of patients with bladder cancer in Yazd, central province of Iran. Int J Cancer Manag. 2018;11(5). doi: $10.5812 / \mathrm{ijcm}$

48. Somi MH, Farhang S, Mirinezhad SK, Naghashi S, Seif-Farshad M, Golzari M. Cancer in East Azerbaijan, Iran: results of a population-based cancer registry. Asian Pac J Cancer Prev. 2008;9 (2):327-330.

49. Akbari ME, Hosseini SJ, Rezaee A, Hosseini MM, Rezaee I, Sheikhvatan M. Incidence of genitourinary cancers in the Islamic Republic of Iran: a survey in 2005. Asian Pac $J$ Cancer Prev. 2008;9(4):549-552.

50. Rohani-Rasaf M, Moradi-Lakeh M, Ramezani R, Asadi-Lari M. Measuring socioeconomic disparities in cancer incidence in Tehran, 2008. Asian Pac J Cancer Prev. 2012;13(6):2955-2960. doi:10.7314/ APJCP.2012.13.6.2955

51. Ahmadi A, Salehi F. Evaluation of observed and the expected incidence of common cancers: an experience from Southwestern of Iran, 2010-2014. J Res Med Sci. 2018;23. doi:10.4103/jrms. JRMS_788_17

52. Shahesmaeili A, Afshar RM, Sadeghi A, Bazrafshan A. Cancer incidence in Kerman Province, Southeast of Iran: report of an ongoing population-based cancer registry, 2014. Asian Pac J Cancer Prev. 2018;19(6):1533.

53. Akbarzadeh PA, Mohammadi KH, ALIZADEH NR, Hajian K. The incidence of bladder cancer in Mazandaran province for three years. JBUMS. 2012;14(3):80-84.

54. Amori N, Aghajani M, Asgarian F, Jazayeri M. Epidemiology and trend of common cancers in Iran (2004-2008). Eur J Cancer Care. 2017;26(5):e12449. doi:10.1111/ecc.2017.26.issue-5

55. Babaei M, Jaafarzadeh H, Sadjadi A, et al. Cancer incidence and mortality in Ardabil: report of an ongoing population-based cancer registry in Iran, 2004-2006. Iran J Public Health. 2009;35-45.

56. Basiri A, Shakhssalim N, Jalaly NY, Miri HH, Partovipour E, Panahi MH. Difference in the incidences of the most prevalent urologic cancers from 2003 to 2009 in Iran. Asian Pac J Cancer Prev. 2014;15(3):1459-1463. doi:10.7314/APJCP.2014.15.3.1459 
57. Haghdoost AA, Baneshi MR, Haji-Maghsoodi S, Molavi-Vardanjani H, Mohebbi E. Application of a network scale-up method to estimate the size of population of breast, ovarian/ cervical, prostate and bladder cancers. Asian Pac J Cancer Prev. 2015;16(8):3273-3277. doi:10.7314/APJCP.2015.16.8.3273

58. Rafiemanesh H, Zahedi A, Mehtarpour M, et al. Cancer epidemiology and trends in North Khorasan Province of Iran. Clin Epidemiol Glob Health. 2018;6(2):51-55. doi:10.1016/j.cegh.2018.02.001

59. Rafiemanesh H, Mehtarpoor M, Mohammadian-Hafshejani A, Salehiniya H, Enayatrad M, Khazaei S. Cancer epidemiology and trends in Sistan and Baluchestan province, Iran. Med J Islam Repub Iran. 2015;29:254.

60. Zahedi A, Rafiemanesh H, Enayatrad M, Ghoncheh M, Salehiniya H. Incidence, trends and epidemiology of cancers in north west of Iran. Asian Pac J Cancer Prev. 2015;16(16):7189-7193. doi:10.7314/ APJCP.2015.16.16.7189

61. Sadjadi A, Malekzadeh R, Derakhshan MH, et al. Cancer occurrence in Ardabil: results of a population-based cancer registry from Iran. Int J Cancer. 2003;107(1):113-118. doi:10.1002/(ISSN)1097-0215

62. Sadjadi A, Zahedi M, Nouraie M, et al. The first population-based cancer survey in Kerman Province of Iran. Iran J Public Health. 2007;36(4):26-34.

63. Somi MH, Dolatkhah R, Sepahi S, et al. Cancer incidence in the East Azerbaijan province of Iran in 2015-2016: results of a populationbased cancer registry. BMC Public Health. 2018;18(1):1266. doi:10.1186/s12889-018-6119-9

64. Salehiniya H, Dashdebi SG, Rafiemanesh H, MohammadianHafshejani A, Enayatrad M. Time trend analysis of cancer incidence in Caspian Sea, 2004-2009: a population-based cancer registries study (Northern Iran). Caspian J Intern Med. 2016;7(1):25.

65. Talaiezadeh A, Tabesh H, Sattari A, Ebrahimi S. Cancer incidence in southwest of iran: first report from khuzestan population-based cancer registry, 2002-2009. Asian Pac J Cancer Prev. 2013;14 (12):7517-7522. doi:10.7314/APJCP.2013.14.12.7517

66. Fararouei M, Parisai Z, Farahmand M, Haghighi RE, Toori MA. Cancer incidence appears to be rising in a small province in Islamic Republic of Iran: a population-based cohort study. East Mediterr Health J. 2015;21(5):319. doi:10.26719/2015.21.5.319

67. Keyghobadi N, Rafiemanesh H, Mohammadian-Hafshejani A, Enayatrad M, Salehiniya H. Epidemiology and trend of cancers in the province of Kerman: Southeast of Iran. Asian Pac J Cancer Prev. 2015;16(4):1409-1413. doi:10.7314/APJCP.2015.16.4.1409

68. Mohagheghi S, Mousavi JS, Malekzadeh R, Parkin M. Cancer incidence in Tehran metropolis: the first report from the Tehran population-based cancer registry, 1998-2001. Arch Iran Med. 2009;12(1):15-23.
69. Masoompour SM, Lankarani KB, Honarvar B, Tabatabaee SH, Moghadami M, Khosravizadegan Z. Changing epidemiology of common cancers in Southern Iran, 2007-2010: a cross sectional study. PLoS One. 2016;11(5):e0155669. doi:10.1371/journal.pone.0155669

70. Vakili M, Pirdehghan A, Adimi M, Sadeghian M, Akhondi M. Epidemiology and trend of cancer in Yazd, a central province of Iran, 2005-2009. J Res Health Sci. 2014;14(3):210-213.

71. Yavari P, Sadrolhefazi B, Mohagheghi M, et al. An epidemiological analysis of cancer data in an Iranian hospital during the last three decades. Asian Pac J Cancer Prev. 2008;9(1):145-150.

72. Rahimi Z, Kasraei R, Najafi F, et al. Cancer notification at a referral hospital of Kermanshah, Western Iran (2006-2009). Asian Pac J Cancer Prev. 2015;16(1):133-137. doi:10.7314/APJCP.2015.16.1.133

73. Rafiemanesh H, Rajaei-Behbahani N, Khani Y, Hosseini S. Incidence trend and epidemiology of common cancers in the center of Iran. Glob J Health Sci. 2016;8(3):146. doi:10.5539/gjhs.v8n3p146

74. Rafiemanesh H, Lotfi Z, Bakhtazad S, Ghoncheh M, Salehiniya H. The epidemiological and histological trend of bladder cancer in Iran J Cancer Res Ther. 2018;14(3):532. doi:10.4103/0973-1482.172134

75. Enayatrad M, Mirzaei M, Salehiniya H, Reza M. Trends in incidence of common cancers in Iran. Asian Pac J Cancer Prev. 2016;17 (S3):39-42. doi:10.7314/APJCP.2016.17.S3.39

76. Koohi F, Salehiniya $H$. The trend of incidence of bladder cancer in Iran, 2003-2009. Urmia Med J. 2015;26(1):1-9.

77. Alireza S, Mehdi N, Ali M, Alireza M, Reza M, Parkin D. Cancer occurrence in Iran in 2002, an international perspective. Asian Pac $J$ Cancer Prev. 2005;6(3):359.

78. Mahdavi S, Amoori N, Salehiniya H, Almasi Z, Enayatrad M. Trend of bladder cancer mortality in Iran (2006 to 2010). Int J Epidemiol Res. 2015;2(4):184-189.

79. Nikfarjam Z, Massoudi T, Salehi M, Salehi M, Khoshroo F. Demographic survey of four thousand patients with 10 common cancers in North Eastern Iran over the past three decades. Asian Pac J Cancer Prev. 2014;15(23):10193-10198. doi:10.7314/APJCP.2014.15.23.10193

80. Farahmand M, Khadem AF, Medhati M, Shokrpour N, Joulaei H, Mehrbani D. Trend of bladder cancer in Fars province, southern Iran, 2002-2006. Iran Red Crescent Med J. 2009;11(4):470-472.

81. Roshani Z, Akbari AK, Shati M, Sahaf R. Prevalence of types of cancers in the elderly covered by insurance of the Islamic Republic of Iran Broadcasting Company in 2015-comparison with younger groups. Asian Pac J Cancer Prev. 2016;17(S3):269-273. doi:10.7314/APJCP.2016.17. S3.269
Research and Reports in Urology

\section{Publish your work in this journal}

Research and Reports in Urology is an international, peer-reviewed, open access journal publishing original research, reports, editorials, reviews and commentaries on all aspects of adult and pediatric urology in the clinic and laboratory including the following topics: Pathology, pathophysiology of urological disease; Investigation and treatment of urological disease; Pharmacology of drugs used for the treatment of urological disease. The manuscript management system is completely online and includes a very quick and fair peer-review system, which is all easy to use. Visit http://www.dovepress.com/ testimonials.php to read real quotes from published authors. 